\title{
Preoperative intensity-modulated chemoradiation therapy with simultaneous integrated boost in rectal cancer: 2-year follow-up results of phase II study
}

\author{
Jasna But-Hadzic, Vaneja Velenik \\ Department of Radiotherapy, Institute of Oncology Ljubljana, Ljubljana, Slovenia
}

Radiol Oncol 2018; 52(1): 23-29.

Received: 8 September 2017

Accepted: 17 September 2017

Correspondence to: Assoc. Prof. Vaneja Velenik, M.D., Ph.D., Institute of Oncology Ljubljana, Zaloška 2, SI-1000 Ljubljana, Slovenia. Phone: +386 15879 503; Fax: +386 15879 416; E-mail: jbut@onko-i.si

Disclosure: No potential conflicts of interest were disclosed.

\begin{abstract}
Background. The aim of the study was to investigate the feasibility and safety of experimental fractionation using intensity modulated radiation therapy with a simultaneous integrated boost (IMRT-SIB) to shorten the overall treatment time without dose escalation in preoperative radiochemotherapy of locally advanced rectal cancer.

Patients and methods. Between January 2014 and November 2015, a total of 51 patients with operable stage II-III rectal adenocarcinoma were treated. The preoperative treatment with intensity modulated radiation therapy (IMRT) and a pelvic dose of $41.8 \mathrm{~Gy}$ and simultaneously delivered $46.2 \mathrm{~Gy}$ to T2/3 and $48.4 \mathrm{~Gy}$ to T4 tumour in 22 fractions, with standard concomitant capecitabine, was completed in 50 patients out of whom 47 were operated. The median follow-up was 35 months.

Results. The rate of acute toxicity $G \geq 3$ was $2.4 \%$. The total downstaging rate was $89 \%$ and radical resection was achieved in $98 \%$ of patients. Pathologic complete response (PCR) was observed in $25.5 \%$ of patients, with 2 -year local control (LC), disease free survival (DFS), and overall survival (OS) of 100\% for this patient group. An intention-to-treat analysis revealed $\mathrm{pN}$ to be a significant prognostic factor for DFS and OS ( $P=0.005$ and 0.030 , respectively). $L C$ for the entire group was 100\%, and 2-year DFS and OS were 90\% (95\% Cl 98.4-81.6) and 92.2\% (95\% Cl 99.6-84.7), respectively. Conclusions. The experimental regime in this study resulted in a high rate of PCR with a low acute toxicity profile. Excellent early results translated into encouraging 2-year LC, DFS, and OS.
\end{abstract}

Key words: rectal cancer; intensity modulated radiation therapy; simultaneous integrated boost; preoperative radiochemotherapy; acute toxicity; pathologic complete response

\section{Introduction}

With standard preoperative treatment of locally advanced rectal cancer (LARC), we can achieve excellent local control; but long term survival is still poor due to a high rate of distant metastases. ${ }^{1,2}$ To target distant microscopic disease, an additional drug has been added to the preoperative treatment in several studies, but with conflicting results of treatment outcome and high acute toxicity. ${ }^{3-5}$
Since dosimetric studies on preoperative intensity-modulated radiotherapy (IMRT) showed a better sparing of organs at risk compared with standard 3-dimensional conformal radiotherapy (3D CRT) in rectal cancer ${ }^{6-9}$, this novel radiation technique has been used in several prospective phase II studies with the aim of improving the treatment outcome in LARC. The treatment intensification consisted of a dose escalation with a simultaneous integrated boost (SIB), with or without the use of 
an additional drug alongside standard concomitant capecitabine. ${ }^{10-15}$ Researchers report an encouraging rate of pathologic complete response (pCR) and local control (LC), but with substantial acute toxicity with oxaliplatin addition ${ }^{12}$ and non-negligible late toxicity with dose escalation. ${ }^{11}$

Because of a promising impact on clinical outcome, but, conflicting toxicity results with dose escalation in preoperative treatment of LARC, we conducted a phase II trial, testing the experimental fractionation with the use of IMRT-SIB in order to shorten the overall treatment time with a biologically effective dose (BED) similar to the one used in standard 3D CRT. In recently published early results from our trial, we demonstrated that preoperative radiochemotherapy with IMRT-SIB without dose escalation, concomitantly with capecitabine, can achieve a high rate of $\mathrm{pCR}$, a downstaging with a very low acute toxicity profile, and excellent compliance. ${ }^{16}$ After the 2-year follow-up, we analysed the impact of experimental fractionation on $\mathrm{LC}$, disease-free survival (DFS), and overall survival (OS).

\section{Patients and methods}

\section{Study design and inclusion criteria}

The trial design, eligibility criteria, and treatment details have been published previously in detail. ${ }^{16}$ In brief, patients had to present with histologically confirmed, operable adenocarcinoma, located within $15 \mathrm{~cm}$ from the anal verge. Patients with locally advanced, non-metastatic disease ( $\mathrm{cT} \geq 3$ and/ or $\mathrm{cN} \geq 1$ on magnetic resonance imaging (MRI) and $\mathrm{M} 0$ on CT thorax/abdomen) without contraindications for chemotherapy were included.

TABLE 1. Biologic effective dose (BED) comparison for standard 3-dimensional conformal radiotherapy (3D CRT) and intensity modulated radioation therapy with simultaneous boost (IMRT-SIB) as experimental fractionation

\begin{tabular}{lccc}
\hline Treatment & $\begin{array}{c}\text { Pelvis } \\
\text { TD/d/BED (Gy) }\end{array}$ & $\begin{array}{c}\text { Tumour T } \leq 3 \\
\text { TD/d/BED (Gy) }\end{array}$ & $\begin{array}{c}\text { Tumour T4 } \\
\text { TD/d/BED (Gy) }\end{array}$ \\
\hline 3D CRT & $45 / 1.8 / \mathbf{3 7 . 5}$ & $50.4 / 1.8 / \mathbf{4 0 . 9}$ & $54 / 1.8 / \mathbf{4 3 . 9}$ \\
IMRT-SIB & $41.8 / 1.9 / \mathbf{3 5 . 9}$ & $46.2 / 2.1 / \mathbf{4 2 . 1}$ & $48.4 / 2.2 / \mathbf{4 5 . 2}$ \\
\hline
\end{tabular}

BED is calculated as BED $=\operatorname{TD} \times(d+a / \beta) /(2+a / \beta)-(T-t) \times D_{\text {prolit }}$ in which TD is the total dose $\mathrm{d}$ dose (Gy) per fraction, $a / \beta$ is the common linear-quadratic quotient (set to $10 \mathrm{~Gy}$ ), $D_{\text {prolif }}$ is the dose recovered due to proliferation (set to $0.6 \mathrm{~Gy} /$ day), $\mathrm{T}=$ total treatment time and $t=$ initia delay time (days, set to 7 days)data from 36 prospective studies, 7 retrospective studies and 17 other articles were used. A total of 131 scientific articles are included, involving 25351 patients. The results were compared with those of a similar overview from 1996 including 15042 patients. The conclusions reached can be summarized thus: The results after rectal cancer surgery have improved during the past decade. It is likely that local failure rates after 5 years of follow-up at hospitals adopting the TME-concept (TME = total mesorectal excision. ${ }^{20}$ Standard fractionation for preoperative rectal cancer treatment with 3D CRT consists of $45 \mathrm{~Gy}$ in 25 fractions to the tumour and regional lymph nodes (pelvis) and additional boost $3 \times 1.8$ Gy (TD 50.4 Gy) in T $\leq 3$ and $5 \times 1.8$ Gy (TD 54 Gy) in T4 tumour.
Prior to treatment, all patients received detailed oral and written information, and signed an informed consent form. The trial was approved by the National Medical Ethics Committee of the Republic of Slovenia (No. 41/12/13) and was in agreement with the Declaration of Helsinki. The study was registered in the ClinicalTrials.gov database (NCT02268006).

\section{Treatment protocol}

The target volumes and dose prescription were defined according to ICRU Reports 50, 62 ${ }^{17}$, and $83 .^{18}$ The gross tumour volume (GTV) encompassed all visible primary tumours. GTV $+1 \mathrm{~cm}$ represented a boost volume (CTV2), and the clinical target volume 1 (CTV1) contained CTV2, mesorectum, and regional lymph nodes from L5/S1 to $4 \mathrm{~cm}$ below the tumour or musculus levator ani. The nodes along arteria iliaca externa were included in case of substantial genitourinary structure infiltration, and the ishiorectal fossa and anal canal in the case of musculus levator ani or anal canal involvement. The internal target volume (ITV) extended up to $0.5 \mathrm{~cm}$ anteriorly in the lower half and up to $1.5 \mathrm{~cm}$ anteriorly in the upper half of the mesorectum. ${ }^{19}$ The planning target volume (PTV) was extended from ITV for $7 \mathrm{~mm}$ posteriorly and laterally, and $10 \mathrm{~mm}$ in other directions.

PTV 1 received 41.8 Gy in 22 fractions and SIB was prescribed to tumour (PTV 2) concomitantly to doses of 46.2 Gy and 48.4 Gy to $\mathrm{T} \leq 3$ and $\mathrm{T} 4$ tumours in 22 fractions, respectively, 5 times per week (Monday to Friday) (Table 1). The main constraints for organs at risk were: $\mathrm{V}_{45 \mathrm{~Gy}}<195 \mathrm{cc}$ and $\mathrm{D}_{\max } \leq 50 \mathrm{~Gy}$; anal canal $\mathrm{V}_{40 \mathrm{~Gy}} \leq 40 \%$ and $\mathrm{D}_{\max } \leq 55$ Gy; iliac crests $\mathrm{V}_{30 \mathrm{~Gy}}<50 \%, \mathrm{~V}_{40 \mathrm{~Gy}}<35 \%$; bladder $\mathrm{V}_{30 \mathrm{~Gy}}<50 \%$ and $\mathrm{V}_{35 \mathrm{~Gy}}<35 \%$; and penile bulb $\mathrm{D}_{90 \%}<$ 50 Gy [16] (Figure 1).

The treatment was delivered on Clinac 2100 CDI (Varian, Palo Alto, USA) using the dynamic multileaf collimator technique with $6 \mathrm{MV}$ photons and a daily position verification (ExacTrac $X$-ray 6D system, BrainLAB AG, Feldkirchen, Germany).

Patients received concomitant chemotherapy with capecitabine from the first to last day of the radiation treatment (including weekends) at a daily dose of $825 \mathrm{mg} / \mathrm{m}^{2} / 12 \mathrm{~h}$. One dose was taken 1 hour prior to irradiation. The treatment compliance and acute toxicity were evaluated weekly according to the common terminology criteria for adverse events (CTCAE) v.4.0. ${ }^{21}$

Surgery was scheduled 6-8 weeks after the completion of chemoradiotherapy, pathologic stage 


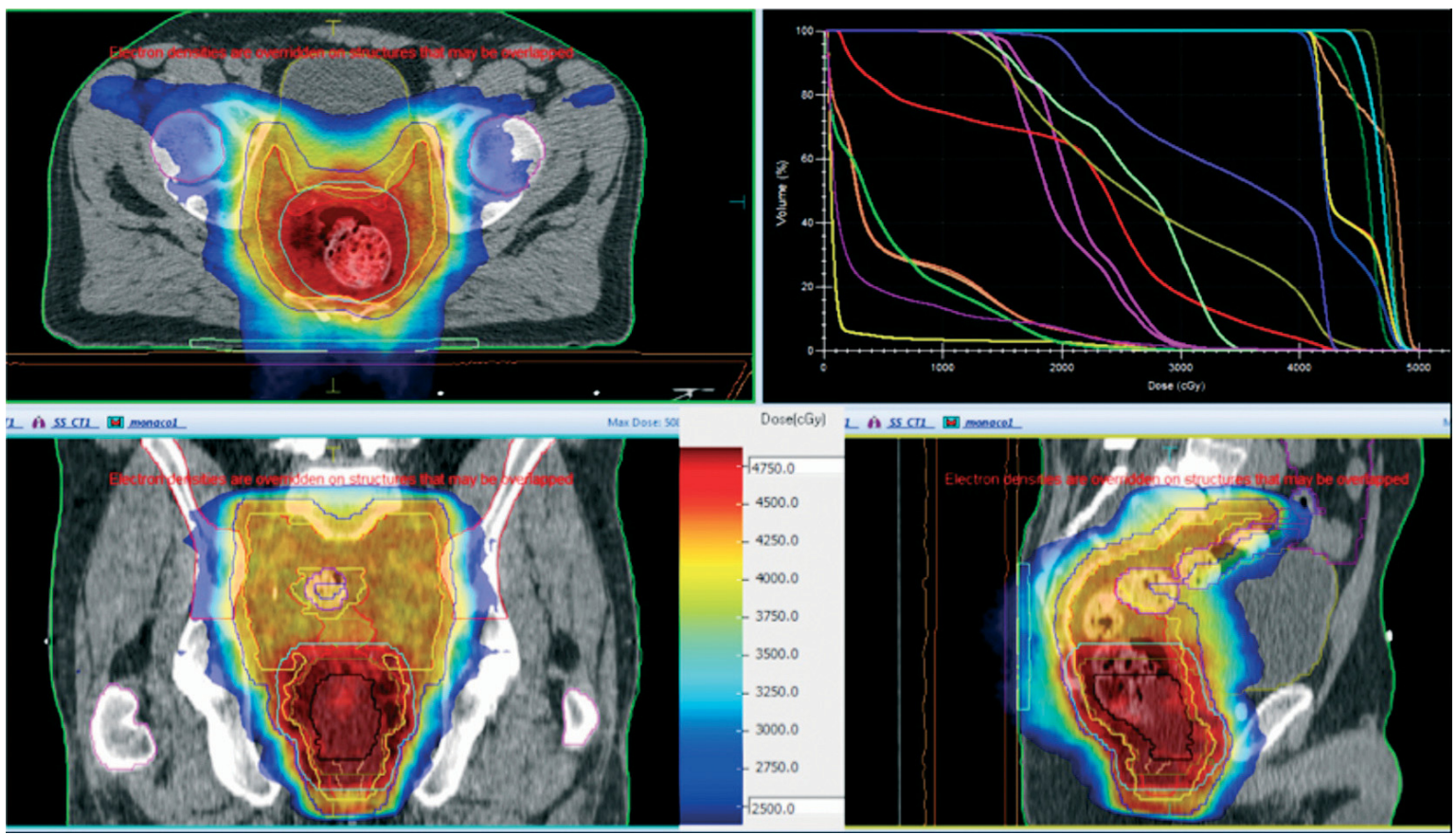

FIGURE 1. Intensity modulated radiation therapy plan met the planning goals.

recorded according to the AJCC $7^{\text {th }}$ edition ${ }^{22}$, and tumour regression grade according to the criteria by Dworak et al. ${ }^{23}$

Six cycles of adjuvant chemotherapy with capecitabine were offered to patients with residual tumour on pathologic examination. After treatment, the follow-up consisted of clinical and serum CEA evaluation every 3 months for two years, and later on a bi-annual basis with abdominal ultrasound every 6 months and a chest radiograph annually.

\section{Statistics}

This was a prospective phase II study on patients with locally advanced rectal cancer, designed to evaluate the pathologic complete response after experimental preoperative treatment as a primary endpoint. The key secondary endpoints were to evaluate the acute toxicity of preoperative treatment, tumour response, LC, DFS, and OS. Late toxicity and the quality of life will be analysed after a 5-year follow-up.

All time intervals were calculated from the date of operation or date of chemoradiotherapy completion (for non-operated patients). The end dates for time calculations were the dates of the last followup or death for OS, and for DFS the dates of detected local/distant relapse, last follow-up, or death. In the patient with primary lung metastasis and in non-operated patients, we counted the DFS time as 0 months.

A statistical analysis was performed using the Statistical Package for the Social Sciences, version 22.0 (SPSS Inc., Chicago, IL, USA). Descriptive statistics were used for presenting general data. Patients surgically treated after chemoradiotherapy completion $(\mathrm{N}=47)$ entered treatment response analysis. Fisher's exact test was used for tumour regression grade prognostic group comparison. The survival curves were calculated with the KaplanMaier method and the influence of the possible prognostic factors on survival was verified by means of the log-rank test.

\section{Results}

Between January 2014 and November 2015, a total of 51 patients were treated (Figure 2). Patients and tumour characteristics were described in detail elsewhere ${ }^{16}$, but, briefly - the median age of the group was 66 years (range, 30-81 years) and two thirds were men. Nearly half of the tumours were located in the lower third of the rectum and 20 patients had positive mesorectal fascia (MRF+). According to AJCC $7^{\text {th }}$ edition ${ }^{22}$, the clinical stages of the disease were as follows: T2N1M0 $(\mathrm{n}=1)$, T3N0M0 $(\mathrm{n}=6)$, T3N1M0 $(\mathrm{n}=15)$, T3N2M0 $(\mathrm{n}=$ 22), T4N1M0 $(n=4), T 4 N 2 M 0(n=2)$, and T3N1M1 $(n=1)$. One patient had a small lung lesion prior to 


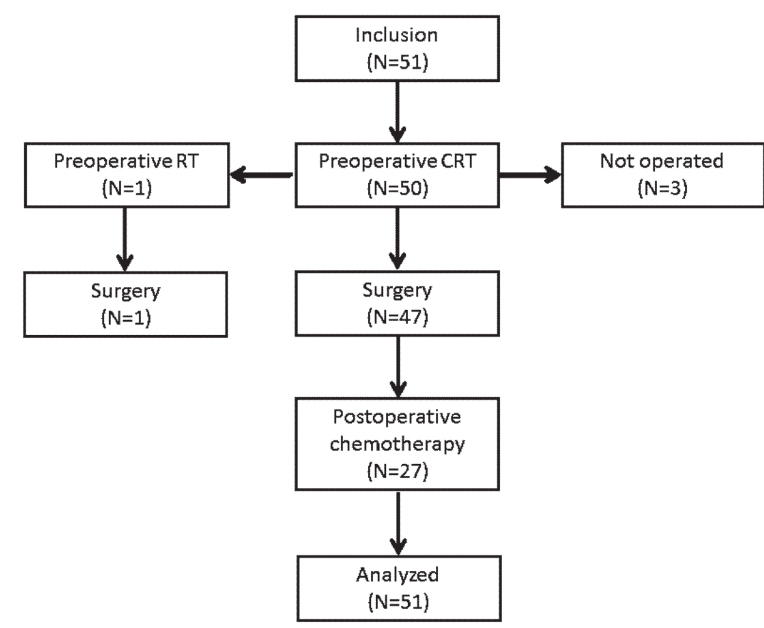

FIGURE 2. Distribution of patients through the trial.

$\mathrm{CRT}$ = radiochemotherapy; $\mathrm{RT}$ = radiotherapy

treatment, but control CT following the treatment revealed a primary metastatic disease with lung metastasis in his case.

\section{Treatment}

Preoperative radiochemotherapy according to protocol was completed by 50 patients in a median of 31 days (range 29-36 days), and one received preoperative short-course radiotherapy ( 25 Gy in 5 fractions) due to ischemic stroke in the first week of experimental treatment. The acute toxicity of

TABLE 2. Influence of potential prognostic factors on overall survival (OS) and disease free survival (DFS)

\begin{tabular}{|c|c|c|}
\hline Prognostic factor & OS & DFS \\
\hline Age & ns & ns \\
\hline Gender & ns & ns \\
\hline $\begin{array}{l}\text { WHO PS } \\
\text { Tumour grade }\end{array}$ & $\begin{array}{l}\text { ns } \\
\text { ns }\end{array}$ & $\begin{array}{l}\text { ns } \\
\text { ns }\end{array}$ \\
\hline cTumour stage $^{a}$ & ns & ns \\
\hline cNodal stage ${ }^{a}$ & ns & ns \\
\hline $\begin{array}{l}\text { TRG } \\
\text { TRG prognostic group } \\
\text { pTumour staged } \\
\text { pNodal stage }\end{array}$ & 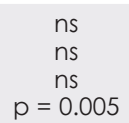 & 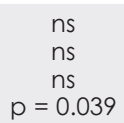 \\
\hline $\begin{array}{l}\mathrm{PCR}^{\mathrm{f}} \\
\text { Adjuvant chemotherapyg } \\
5-6 \text { vs. } \leq 4 \text { cycles* }\end{array}$ & $\begin{array}{c}n s \\
p=0.009\end{array}$ & $\begin{array}{c}n s \\
p=0.012\end{array}$ \\
\hline
\end{tabular}

TRG = tumour regression grade $23 ;$ WHO PS $=$ WHO performance status: $\mathrm{f}$ - pathologic complete response; $\mathrm{g}$ - chemotherapy; * calculated for 36 patients with indication for adjuvant chemotherapy; ns = not specific $(p>0.05)$.

a according the AJCC, 7th edition ${ }^{22}$ preoperative treatment was mild, with only two G3 acute adverse events with infectious enterocolitis and radiodermatitis.

Surgery was performed in 48 patients and operation was omitted in three due to the patient's refusal, metachronous pancreatic carcinoma, and serious bleeding from rectal varices. Low anterior resection was performed in 40 patients, abdominoperineal resection in 7 , and pelvic exenteration in 1 . Radical resection (R0) was achieved in 47 patients and one had a microscopic carcinoma focus in the circumferential margin (R1). Major complications (CTCAE v.4.0 G $\geq 3$ ) occurred in 4 out of 48 patients. A rescue surgery with pelvic exenteration was performed in the patient with rectal varices due to tumour progression 35 months after chemoraditherapy completion. She is disease-free 4 months after R0 resection.

Adjuvant chemotherapy was given to patients who did not achieve pCR. In four patients, adjuvant treatment was omitted due to preoperative adverse events (ischemic stroke in two patients and infectious enterocolitis G3 in one), and one patient refused it.

\section{Treatment response}

Among 47 operated patients who completed preoperative treatment according to protocol, 12 achieved pathologic complete response (25.5\%). The total downstaging rate was $89 \%$ (42 of 47 patients), with a decrease in $\mathrm{T}$ and $\mathrm{N}$ stages observed in $32(68 \%)$ and in $39(83 \%)$ patients, respectively. According to the Dworak criteria ${ }^{23}$, the tumour regression grades (TRG) were TRG 4, TRG 3, TRG 2, TRG 1 , and TRG 0 in 12,16, 13, 6, and 0 patients, respectively.

\section{Survival}

An intention-to-treat analysis was performed on all 51 patients. In the median follow-up time of 35 months (range, 14-43 months), we recorded no local relapses and 4 distal relapses (two patients with lung metastases and two with both liver and paraaortic lymph node metastases). To date, 44 patients are alive without rectal cancer; two patients are alive with primary disease (one with an intact primary tumour and one with liver metastases). Three patients have died because of primary rectal cancer disease and two of other causes (myocardial infarction and pancreatic cancer).

Cumulative 2-year LC, DFS, and OS were 100\%, 90\% (95\% CI 98.4-81.6), and 92.2\% (95\% CI 99.6- 
TABLE 3. Comparison of tumour regression grade in patients with RO resection

\begin{tabular}{lcccccc} 
& $\begin{array}{c}\text { IMRT-SIB But-Hadzic et al. } \\
\mathbf{N}=\mathbf{4 6}\end{array}$ & $\begin{array}{c}\text { 3D CRT Focas et al. } \\
\mathbf{N}=\mathbf{3 2}\end{array}$ & $\mathbf{p}$ & $\begin{array}{c}\text { IMRT-SIB But-Hadzic et al. }{ }^{16} \\
\mathbf{N}=\mathbf{4 6}\end{array}$ & $\begin{array}{c}\text { IMRT-SIB Li et al. }{ }^{10} \\
\mathbf{N}=\mathbf{5 8}\end{array}$ \\
\hline TRG 4 & $12(26 \%)$ & $40(10 \%)$ & $\mathbf{0 . 0 0 4}$ & $12(26 \%)$ & $19(33 \%)$ & 0.302 \\
TRG 2-3 & $29(63 \%)$ & $254(66 \%)$ & 0.404 & $29(63 \%)$ & $20(35 \%)$ & 0.003 \\
TRG 0-1 & $5(11 \%)$ & $91(24 \%)$ & $\mathbf{0 . 0 3 1}$ & $5(11 \%)$ & $19(32 \%)$ & 0.007 \\
\hline
\end{tabular}

3D CRT = 3D conformal radiotherapy; IMRT-SIB = intensity modulated radiation therapy with simultaneous boost; TRG = tumour regression grading ${ }^{23}$

84.7), respectively. The possible influence of potential prognostic factors on OS and DFS was determined by means of the log-rank test (Table 2). There was no link between gender, age, performance status, cT, cN, pT, or TRG and survival. Patients with pN2 had significantly worse OS and DFS (Figure 3). In the group of 36 patients that had an indication for adjuvant chemotherapy, we found that the patients who received 5-6 cycles of chemotherapy had significantly better OS and DFS compared with $\leq 4$ cycles of chemotherapy (Figure 3 ). We found a trend toward different OS for patients in different TRG prognostic group, although nonsignificant. Two-year OS's for good (TRG 4), intermediate (TRG 2-3,) and bad (TRG 0-1) prognostic groups were $100 \%, 93.3 \%$, and $83.3 \%$, respectively $(p=0.426)$. Local control, 2-year OS, and 2-year DFS were all $100 \%$ for 12 patients with pCR.

\section{Discussion}

The main limiting factor in the preoperative treatment of locally advanced rectal cancer is acute toxicity - mainly gastrointestinal - which has been preventing the intensification of standard radiochemotherapy for rectal cancer in the last decade. To date, only few prospective studies have used the dosimetric advantage of IMRT-SIB for preoperative treatment intensification of locally advanced rectal cancer. ${ }^{10-15}$ With our experimental preoperative fractionation regime without dose escalation, with standard capecitabine, we report lower acute toxicity rates and comparable treatment results to these dose-escalated studies.

In a previous publication, we reported a very low acute toxicity profile with only $2.4 \%$ G3 acute toxicities ${ }^{16}$, which is lower than two comparable studies with capecitabine. In a Chinese study, 41.8 Gy was delivered to an elective volume in 22 fractions and the tumour-involved lymph nodes received 50.6 Gy. ${ }^{10}$ The pelvis received 46 Gy in 23 fractions in a Spanish study with simultaneous
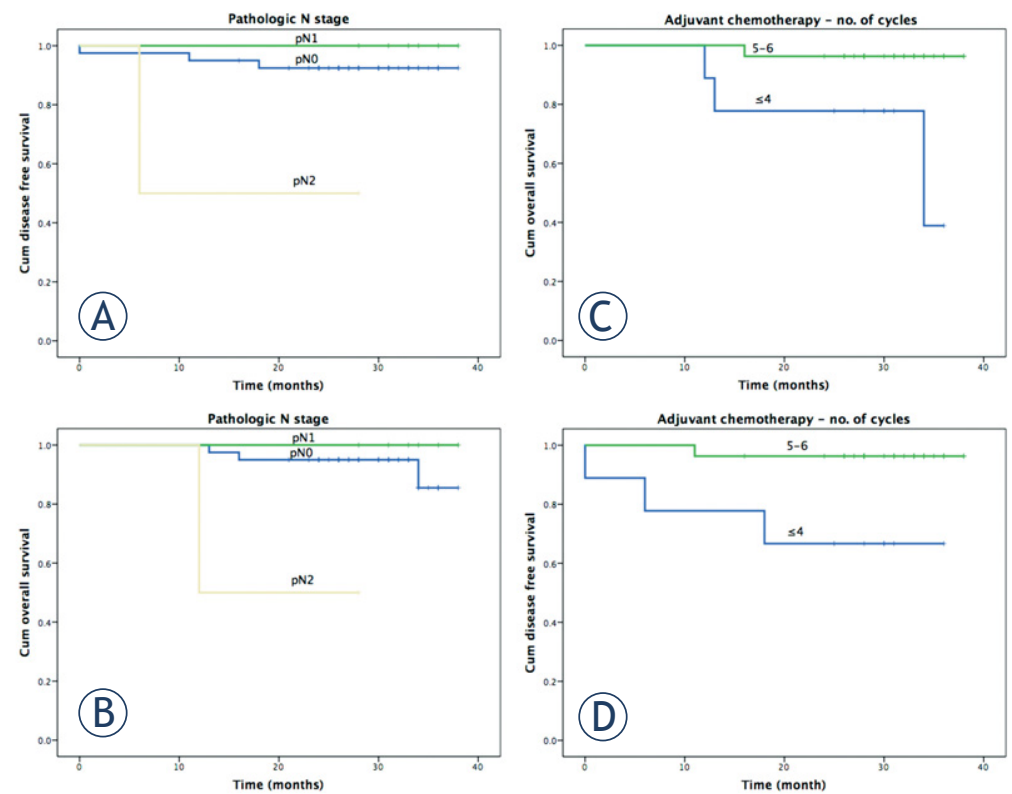

FIGURE 3. Prognostic significance of pathologic nodal stage $(\mathrm{pN})$ on 2-year disease-free survivala, overall survival ${ }^{b}$, prognostic significance of the received number of adjuvant chemotherapy cycles in patients without PCR on 2-year disease-free survivalc, and overall survivald in rectal cancer after preoperative radiochemotherapy and surgery.

dose escalation to $57.5 \mathrm{~Gy}$ to macroscopic disease. ${ }^{12}$ Authors reported $14 \%$ and $7.6 \% \mathrm{G} \geq 3$ acute toxicity rates, respectively. In two drug concomitant chemotherapy (oxaliplatin/capecitabine) dose-escalation trials, the toxicity rates were even higher, up to $44.4 \% .^{13-15}$

The shorter treatment time in our trial resulted in $25.5 \% \mathrm{pCR}$ and excellent downstaging rates of $68 \%$ and $83 \%$ for $\mathrm{T}$ and $\mathrm{N}$ stages, respectively. In our historic cohort with 3D CRT rates of pCR, T, and $\mathrm{N}$ downstaging were $9 \%, 40 \%$, and $52.9 \%$, respectively. ${ }^{24}$ Our pCR rate did not significantly differ from the $31 \%$ and $30,6 \%$ rates in the Chinese and Spanish trials, but was significantly higher compared to our historic cohort.

We observed improved results with a BED similar to standard preoperative treatment. Because of a strong positive correlation between $\mathrm{pCR}$ and the 
dose of radiation ${ }^{25}$, we believe that there are multiple factors positively influencing the results of our trial. Firstly, if the time factor in our calculations is underestimated due to a short lag-period ${ }^{26}$, the BED of our fractionation regime is higher and improvement is achieved due to a steep dose response curve. Secondly, in historic 3D CRT trials, pretreatment pelvic MRI was not mandatory ${ }^{27}$ and the clinical stage was unreliable. Even in the era of MRI, only recently has $\mathrm{cN}$ begun being determined according to morphology. ${ }^{28}$ Thirdly, there was a huge improvement in the precision of the radiotherapy process in recent years. In our study protocol, we tried to minimize the dosimetric impact of inter-observer variability ${ }^{29}$ by using detailed contouring guidelines and a co-registered planning $\mathrm{MRI}^{30}$ when available. A non-uniform safety margin was applied and IGRT was used. In our previous 3D CRT trial, the contouring guidelines were more loose and GTV was contoured according to CT, since MRI was done only in $5 \%$ of patients. ${ }^{24} \mathrm{~A}$ uniform $1 \mathrm{~cm}$ safety margin was used, not counting for organ motion, and the patient position was verified with weekly portal films only. Consequently, systematic errors were substantial and could have contributed to poorer results.

Our tumour downstaging rate is comparable to the Chinese trial; but in a Spanish trial, the rate was higher $(76.4 \%)$ with a higher dose escalation. ${ }^{10,12}$ In both studies, an additional boost was applied to the involved lymph nodes with only a 5 $\mathrm{mm}$ margin and position verification with weekly portal films in the Chinese and a daily cone beam CT in the Spanish trial. Our N-downstaging rate is similar to that in the Chinese research and higher than the Spanish trial despite lower BED, which suggests that an additional boost to the involved lymph nodes is not mandatory. Another explanation of these results would be that the $5 \mathrm{~mm}$ margin around the nodal GTV that was used in both of the other trials was not sufficient to adequately cover the affected nodes with the boost dose and the $\mathrm{N}$ downstaging rate could have been higher.

Since the $\mathrm{pCR}$ rate has a poor treatment prognostic value ${ }^{31}$ and the downstaging comparison with historic trials is not reliable, we performed a comparison of three prognostic groups according to late results of $\mathrm{CAO} / \mathrm{ARO} / \mathrm{AIO}-94$ trial. They found a significant impact on 10-year DFS for the good (TRG 4), intermediate (TRG 2-3), and bad (TRG $0-1)$ response groups. We compared the proportions from our study to comparable preoperative studies with concomitant capecitabine (Table 3). In comparison to $3 \mathrm{D} \mathrm{CRT}^{32}$, we achieved a higher
$\mathrm{pCR}$ rate (TRG 4; $\mathrm{p}=0.004)$ and observed less bad responses to treatment (TRG $0-1 ; p=0.031$ ) with an equal proportion of patients in the intermediate prognostic group. In comparison with dose-escalation IMRT-SIB preoperative treatment ${ }^{10}$, we didn't find a significant difference in the good prognostic group, but the proportion of patients with an intermediate response was higher $(p=0.003)$ with fewer patients exhibiting a bad response in our study ( $p$ $=0.007$ ), which could be a consequence of a more precise radiotherapy procedure.

We report an excellent 2-year LC of $100 \%$, and 2-year DFS and OS of $90 \%$ and $92,2 \%$, respectively. The results are comparable to more intensified preoperative treatment regimes with reported 2-3-year LC 70-100\%, DFS 86-95\% and OS 64-96\%. In concordance with other studies, we found $\mathrm{pN}$ to be the main prognostic factor on OS and DFS ${ }^{27}$; no association between pCR and survival; and an excellent prognosis for $\mathrm{pCR}$ group of patients (2-year LC, DFS, and OS all 100\%). The main limitations of our study are the lack of randomization, the small sample size, and no long-term follow-up. Longer follow-up of the patients is needed to determine if excellent early results will translate to improved long-term results, and to determine the impact of our treatment protocol on late toxicity and QoL.

In conclusion high rate of $\mathrm{PCR}$ and downstaging after preoperative treatment of LARC with IMRT-SIB in 22 fractions without dose escalation, concomitant with capecitabine, translated into excellent 2-year LC, DFS, and OS (100\%, 90\%, and $92.2 \%$, respectively). With the presented results, we have confirmed the superiority of our study to the conventional preoperative regimen.,24 Because of similar results to other IMRT trials and lower acute toxicity profile, our experimental regime is eligible for testing treatment intensification with a second drug in order to further improve the treatment efficacy.

\section{References}

1. Bujko K, Michalski W, Kepka L, Nowacki MP, Nasierowska-Guttmejer A Tokar $P$, et al. Association between pathologic response in metastatic lymph nodes after preoperative chemoradiotherapy and risk of distant metastases in rectal cancer: an analysis of outcomes in a randomized trial. Int J Radiat Oncol Biol Phys 2007; 67: 369-77. doi: 10.1016/j.ijrobp.2006.08.065

2. Leibold T, Shia J, Ruo L, Minsky BD, Akhurst T, Gollub M J, et al. Prognostic implications of the distribution of lymph node metastases in rectal cancer after neoadjuvant chemoradiotherapy. J Clin Oncol 2008; 26: 2106-11. doi: 10.1200/JCO.2007.12.7704

3. Gérard JP, Azria D, Gourgou-Bourgade S, Martel-Lafay I, Hennequin C, Etienne PL, et al. Clinical outcome of the ACCORD 12/0405 PRODIGE 2 randomized trial in rectal cancer. J Clin Oncol 2012; 30: 4558-65. doi: 10.1200/ JCO.2012.42.8771 
4. Rödel C, Liersch T, Becker H, Fietkau R, Hohenberger W, Hothorn T, et al. Preoperative chemoradiotherapy and postoperative chemotherapy with fluorouracil and oxaliplatin versus fluorouracil alone in locally advanced rectal cancer: initial results of the German CAO/ARO/AIO-04 randomised phase 3 trial. Lancet Oncol 2012; 13: 679-87. doi: 10.1016/S1470-2045(12)70187-

5. Velenik $V$, Ocvirk J, Music, $M$, Bracko $M$, Anderluh $F$, Oblak I, et al. Neoadjuvant capecitabine, radiotherapy, and bevacizumab (CRAB) in locally advanced rectal cancer: results of an open-label phase II study. Radiat Oncol. 2011; 6: 105. doi: 10.1186/1748-717X-6-105

6. Arbea L, Ramos, LI, Martínez-Monge R, Moreno M, Aristu, J. Intensitymodulated radiation therapy (IMRT) vs. 3D conformal radiotherapy (3DCRT) in locally advanced rectal cancer (LARC): dosimetric comparison and clinical implications, Radiat Oncol 2010; 5: 17. doi: 10.1186/1748-717X-5-17

7. Guerrero Urbano MT, Henrys AJ, Adams EJ, Norman AR, Bedford IL, Harrington KJ, et al. Intensity-modulated radiotherapy in patients with locally advanced rectal cancer reduces volume of bowel treated to high dose levels. Int J Radiat Oncol Biol Phys 2006; 65: 907-16. doi: 10.1016/j. ijrobp.2005.12.056

8. Engels B, De Ridder M, Tournel K, Sermeus A, De Coninck P, Verellen D, et al. Preoperative helical tomotherapy and megavoltage computed tomography for rectal cancer: impact on the irradiated volume of small bowel. Int J Radiat Oncol Biol Phys 2009; 74: 1476-80. doi: 10.1016/j.jijrobp.2008.10.017

9. Mok H, Crane $\mathrm{CH}$, Palmer MB, Briere TM, Beddar S, Delclos ME, et al. Intensity modulated radiation therapy (IMRT): differences in target volumes and improvement in clinically relevant doses to small bowel in rectal carcinoma. Radiat Oncol 2011; 6: 63. doi: 10.1186/1748-717X-6-63

10. Li JL, Ji JF, Cai Y, Li XF, Li YH, Wu H, et al. Preoperative concomitant boost intensity-modulated radiotherapy with oral capecitabine in locally advanced mid-low rectal cancer: a phase II trial". Radiother Oncol 2012; 102: 4-9. doi: 10.1016/j.radonc.2011.07.030

11. Engels B, Platteaux N, Van den Begin R, Gevaert T, Sermeus A, Storme, G, et al. Preoperative intensity-modulated and image-guided radiotherapy with a simultaneous integrated boost in locally advanced rectal cancer: report on late toxicity and outcome. Radiother Oncol 2014; 110: 155-9. doi: 10.1016/j. radonc.2013.10.026

12. Hernando-Requejo O, López M, Cubillo A, Rodriguez A, Ciervide R, Valero J, et al. Complete pathological responses in locally advanced rectal cancer after preoperative IMRT and integrated-boost chemoradiation. Strahlenther Onkol 2014;190: 515-20. doi: 10.1007/s00066-014-0650-0

13. Picardi V, Macchia G, Guido A, Giaccherini L, Deodato F, Farioli A, et al. Preoperative chemoradiation with VMAT-SIB in rectal cancer: a phase II study. Clin Colorectal Cancer 2016. doi: 10.1016/j.clcc.2016.06.004

14. Hong TS, Moughan J, Garofalo MC, Bendell J, Berger AC, Oldenburg NBE, et al. NRG Oncology Radiation Therapy Oncology Group 0822: a phase 2 study of preoperative chemoradiation therapy using intensity modulated radiation therapy in combination with capecitabine and oxaliplatin for patients with locally advanced rectal cancer. Int J Radiat Oncol Biol Phys 2015; 93: 29-36. doi: 10.1016/j.ijrobp.2015.05.005

15. Zhu J, Liu F, Gu W, Lian P, Sheng W, Xu J, et al. "Concomitant boost IMRTbased neoadjuvant chemoradiotherapy for clinical stage II/III rectal adenocarcinoma: results of a phase II study. Radiat Oncol 2014; 9: 70. doi: 10.1186/1748-717X-9-70

16. But-Hadzic J, Anderluh F, Brecelj E, Edhemovic I, Secerov-Ermenc A, Hudej $\mathrm{R}$, et al. Acute toxicity and tumor response in locally advanced rectal cancer after preoperative chemoradiation therapy with shortening of the overall treatment time using intensity-modulated radiation therapy with simultaneous integrated boost: a phase 2 trial. Int J Radiat Oncol Biol Phys 2016; 96: 1003-10. doi: 10.1016/j.jjrobp.2016.08.031

17. ICRU. Prescribing, recording and reporting photon beam therapy (ICRU report 50). ICRU Report 1993: 357-60. doi: 10.2307/3578862

18. Gregoire V, Mackie TR. Dose prescription, reporting and recording in intensity-modulated radiation therapy: a digest of the ICRU Report 83. Imaging Med 2011; 3: 367-73. doi: 10.2217/iim.11.22

19. Van Triest B, Nijkamp J, van Herk M, Sonke JJ, de Jong R, Hollmann B, et al. Repeat CT assessed CTV variation and PTV margins for short- and longcourse pre-operative RT of rectal cancer. Radiother Oncol 2012; 102: 399405. doi: 10.1016/j.radonc.2011.11.011
20. Glimelius B, Gronberg H, Jarhult J, Wallgren A, Cavallin-Stahl E, Grönberg $\mathrm{H}$, et al. A systematic overview of radiation therapy effects in rectal cancer. Acta Oncol 2003; 42: 476-92.

21. U. S. Department of Health and Human Services, National Institutes of Health, National Cancer Institute. Common terminology criteria for adverse events (CTCAE), Version 4.0 (CTCAE). May 28, 2009: Available at: https://www.eortc.be/services/doc/ctc/CTCAE 4.03 2010-06-14 QuickReference_5x7.pdf. [Citated 2017 Jun 15].

22. Edge SB, Compton CC. The American Joint Committee on Cancer: the 7th edition of the AJCC cancer staging manual and the future of TNM. Ann Surg Oncol 2010; 17: 1471-4. doi: 10.1245/s10434-010-0985-4

23. Dworak O, Keilholz L, Hoffmann A. Pathological features of rectal cancer after preoperative radiochemotherapy. Int J Colorectal Dis. 1997; 12: 19-23.

24. Velenik V, Oblak I, Anderluh F. Long-term results from a randomized phase II trial of neoadjuvant combined-modality therapy for locally advanced rectal cancer. Radiat Oncol 2010; 5: 88. doi: 10.1186/1748-717X-5-88

25. Burbach JPM, den Harder AM, Intven $M$, van Vulpen $M$, Verkooijen $\mathrm{HM}$, Reerink O. Impact of radiotherapy boost on pathological complete response in patients with locally advanced rectal cancer: a systematic review and meta-analysis. Radiother Oncol 2014; 113: 1-9. doi: 10.1016/j. radonc.2014.08.035

26. Suwinski R, Taylor JM, Withers HR. Rapid growth of microscopic rectal cancer as a determinant of response to preoperative radiation therapy. Int J Radiat Oncol Biol Phys 1998; 42: 943-51. doi: http://dx.doi.org/10.1016/ S0360-3016(98)00343-5

27. Fokas $E$, Liersch $T$, Fietkau R, Hohenberger W, Beissbarth $T$, Hess $C$, et al. Tumor regression grading after preoperative chemoradiotherapy for locally advanced rectal carcinoma revisited: updated results of the CAO/ARO/AIO94 trial. J Clin Oncol 2014; 32: 1554-62. doi: 10.1200/JCO.2013.54.3769

28. Brown G, Richards CJ, Bourne MW, Newcombe RG, Radcliffe AG, Dallimore NS, et al. Morphologic predictors of lymph node status in rectal cancer with use of high-spatial-resolution MR imaging with histopathologic comparison. Radiology 2003; 227: 371-7. doi: 10.1148/radiol.2272011747

29. Lobefalo F, Bignardi M, Reggiori G, Tozzi A, Tomatis S, Alongi F, et al. Dosimetric impact of inter-observer variability for 3D conformal radiotherapy and volumetric modulated arc therapy: the rectal tumor target definition case. Radiat Oncol 2013; 8: 176. doi: 10.1186/1748-717X-8-176

30. O'Neill BD, Salerno G, Thomas K, Tait DM, Brown G. MR vs CT imaging: low rectal cancer tumour delineation for three-dimensional conformal radiotherapy. Br J Radiol 2009; 82: 509-13. doi: 10.1259/bjr/60198873

31. Glynne-Jones R, Mawdsley S, Pearce T, Buyse M. Alternative clinical end points in rectal cancer - are we getting closer? Ann Oncol 2006; 17: 1239-48. doi: 10.1093/annonc/mdl173

32. Rödel C, Martus P, Papadoupolos T, Füzesi L, Klimpfinger M, Fietkau R, et al. Prognostic significance of tumor regression after preoperative chemoradiotherapy for rectal cancer. J Clin Oncol 2005; 23: 8688-96. doi: 10.1200/ JCO.2005.02.1329 